DOI: https://doi.org/10.11144/Javeriana.umed61-4.migr

\title{
Lo más temido de la migraña: el infarto migrañoso
}

\section{The Most Feared of Migraine: Migranous Infarction}

Recibido: 12/12/2019 | Aceptado: 31/03/2020

\section{Andrés Felipe Cárdenas-Cruz}

Semillero de Neurología Vascular, Servicio de Neurología, Pontificia Universidad Javeriana, Bogotá, Colombia

Julián Santana

Semillero de Neurología Vascular, Servicio de Neurología, Pontificia Universidad Javeriana. Servicio de Neurología, Hospital Universitario San Ignacio, Bogotá, Colombia

María Paula Aguilera-Peña Semillero de Neurología Vascular, Servicio de Neurología, Pontificia Universidad Javeriana, Bogotá,

Colombia

\section{Elza Juliana Coral Casas}

Semillero de Neurología Vascular, Servicio de Neurología, Pontificia Universidad Javeriana. Servicio de Neurología, Hospital Universitario San Ignacio, Bogotá, Colombia

a Autor de correspondencia: acardenasc@javeriana.edu.co

Cómo citar: Cárdenas-Cruz AF, Santana J, AguileraPeña MP, Coral Casas EJ. Lo más temido de la migraña: el infarto migrañoso. Univ. Med. 2020;61(4). https:// doi.org/10.11144/Javeriana.umed61-4.migr

\section{RESUMEN}

El infarto migrañoso se define como el ataque cerebrovascular (ACV) que ocurre durante un episodio de migraña con aura, cuyos síntomas se prolongan durante más de sesenta minutos, se tornan persistentes y en neuroimágenes se relacionan con isquemia cerebral. A pesar de que la fisiopatología subyacente a la migraña como posible causa o factor de riesgo para presentar un ACV no está clara, el vasoespasmo y los cambios en el flujo sanguíneo son mecanismos que pueden explicar este fenómeno. La evidencia acumulada sugiere una asociación entre la migraña con aura y el riesgo de ACV, ataque isquémico transitorio e, incluso, enfermedad coronaria, sin embargo, es bajo el aumento absoluto de riesgo. El artículo presenta el caso de dos mujeres que consultaron al servicio de urgencias con migraña con aura asociado a defectos campimétricos prolongados; en ambos casos se documentaron, mediante resonancia magnética, lesiones isquémicas en el lóbulo occipital.

Palabras clave

ataque cerebrovascular isquémico; cefalea; depresión cortical propagada; migraña con aura; mujeres.

\begin{abstract}
Migrainous infarction is defined as a stroke that occurs during an episode of migraine with aura, in which symptoms extend for more than 60 minutes becoming persistent and related to cerebral ischemia in neuroimaging. Although the pathophysiology of migraine as a possible cause or risk factor for stroke is not clear, vasospasm and changes in
\end{abstract}


blood flow are mechanisms that can explain this phenomenon. The accumulated evidence suggests an association between migraine with aura and the risk of stroke, transient ischemic attack and coronary heart disease, however, the absolute increase in risk is low. We present the case of two women who consulted to the emergency room, with migraine with aura associated with prolonged campimetric defects, in both cases ischemic lesions in the occipital lobe were documented by magnetic resonance imaging.

Keywords

ischemic stroke; headache; cortical spreading depression; migraine with aura; women.

\section{Introducción}

La migraña se considera la cefalea primaria más frecuente en el mundo (1). Afecta hasta el $12 \%$ de la población general y es más frecuente en mujeres en una relación de 3:1 $(2,3)$. Se define como un trastorno episódico, catalogado de intensidad moderada a severa, pulsátil, de 4 a 72 horas de duración y, generalmente, asociado con náuseas o fotofobia y fonofobia (tabla 1) (4). Dentro del espectro de la enfermedad existe la migraña sin aura y con esta. Fisiopatológicamente, la segunda consiste en una alteración focal y transitoria de las neuronas, secundaria a la onda de depresión cortical propagada (DCP) (5). La onda de DCP despolariza desde la corteza posterior hacia el lóbulo frontal, causando disfunción celular en individuos susceptibles. Ello genera una activación aferente del sistema trigeminal y altera la permeabilidad la barrera hematoencefálica mediante la regulación positiva de metaloproteinasas de matriz $(6,7)$.
Tabla 1

Criterios diagnósticos para migraña e infarto migrañoso

\begin{tabular}{|c|c|}
\hline Migraña sin aura & Migraña con aura \\
\hline $\begin{array}{l}\text { A. Al menos cinco crisis que cumplen los } \\
\text { criterios B-D. }\end{array}$ & $\begin{array}{l}\text { A. Al menos dos crisis que cumplen los criterios } \\
\text { B y C. }\end{array}$ \\
\hline $\begin{array}{l}\text { B. Episodios de cefalea de entre } 4 \text { y } 72 \\
\text { horas de duración (no tratados o tratados } \\
\text { sin éxito). }\end{array}$ & $\begin{array}{l}\text { B. Uno o más de los síntomas de aura siguientes } \\
\text { totalmente reversibles: }\end{array}$ \\
\hline $\begin{array}{l}\text { C. La cefalea presenta al menos dos de las } \\
\text { siguientes cuatro características: }\end{array}$ & 1. Visuales. \\
\hline 1. Localización unilateral. & 2. Sensitivos. \\
\hline 2. Carácter pulsátil. & 3. De habla o del lenguaje. \\
\hline 3. Dolor de intensidad moderada o severa. & 4. Motores. \\
\hline $\begin{array}{l}\text { 4. Empeorada por o condiciona el } \\
\text { abandono de la actividad física habitual } \\
\text { (p. ej., andar o subir escaleras). }\end{array}$ & 5. Troncoencefálicos. \\
\hline $\begin{array}{l}\text { D. Al menos uno de los siguientes durante } \\
\text { la cefalea: }\end{array}$ & 6. Retinianos. \\
\hline 1. Náuseas o vómitos. & $\begin{array}{l}\text { C. Al menos dos de las siguientes cuatro } \\
\text { caracteristicas: }\end{array}$ \\
\hline 2. Fotofobia y fonofobia. & $\begin{array}{l}\text { 1. Progresión gradual de al menos uno de los } \\
\text { sintomas de aura durante un periodo } \geq 5 \text { min o } \\
\text { dos o más sintomas se presentan } \\
\text { consecutivamente. }\end{array}$ \\
\hline $\begin{array}{l}\text { E. Sin mejor explicación por otro } \\
\text { diagnóstico de la ICHD-III. }\end{array}$ & $\begin{array}{l}\text { 2. Cada sintoma de aura tiene una duración de } \\
\text { entre } 5 \text { y } 60 \text { minutos. }\end{array}$ \\
\hline & $\begin{array}{l}\text { 3. Al menos uno de los sintomas de aura es } \\
\text { unilateral. }\end{array}$ \\
\hline & $\begin{array}{l}\text { 4. El aura se acompaña o se sigue antes de } 60 \\
\text { minutos, de cefalea. }\end{array}$ \\
\hline & $\begin{array}{l}\text { D. Sin mejor explicación por otro diagnóstico } \\
\text { de la ICHD-III y se ha descartado un ataque } \\
\text { isquémico transitorio. }\end{array}$ \\
\hline \multicolumn{2}{|l|}{ Infarto migrañoso } \\
\hline \multicolumn{2}{|c|}{ A. Un episodio de migraña que cumpla los criterios B y C. } \\
\hline \multicolumn{2}{|c|}{$\begin{array}{l}\text { B. Ocurre en un paciente con migraña con aura y es idéntico a las crisis previas, salvo por } \\
\text { la duración de uno o más síntomas de aura, que persisten más allá de } 60 \text { minutos. }\end{array}$} \\
\hline \multicolumn{2}{|c|}{ C. La neuroimagen revela infarto isquémico en un área congruente. } \\
\hline \multicolumn{2}{|l|}{ D. Sin mejor explicación por otro diagnóstico. } \\
\hline
\end{tabular}

Fuente: Clasificación Internacional

de las Cefaleas, 3.a edición, 2013.

La migraña con aura se presenta en alrededor del $15 \%$ al $20 \%$ de los pacientes con migraña y en su presentación puede simular un ACV. Según la Sociedad Internacional de Cefaleas, las auras típicas de la migraña se desarrollan gradualmente en un periodo de 5 a 60 minutos, tienen una combinación de manifestaciones positivas y negativas, son completamente reversibles y se caracterizan por síntomas visuales, sensitivos o del lenguaje, sin afectación motora (4).

La relación entre migraña y accidente cerebrovascular (ACV) es compleja, ya que ambas patologías son comunes y pueden estar temporalmente relacionadas; sin embargo, metanálisis recientes han descrito una asociación fisiopatológica entre estas dos entidades y han demostrado un ligero aumento en el riesgo absoluto de ACV, así como en el riesgo de presentar un infarto agudo de miocardio $(1,5)$. Incluso se ha encontrado una correlación con la presencia de foramen oval permeable (FOP) y con fibrilación auricular paroxística (8). El infarto migrañoso constituye un reto diagnóstico para 
todos los profesionales de la salud, pues nunca se debe subestimar y siempre se debe sospechar en pacientes que consulten por síntomas de aura que se prolonguen durante más de una hora. A continuación, se presenta el caso de dos mujeres que consultaron al servicio de urgencias del Hospital Universitario San Ignacio en Bogotá, Colombia.

\section{Presentación de los casos}

\section{Caso 1}

El caso corresponde a una mujer de 38 años de edad, natural y procedente de Bogotá, quien ingresó al servicio de urgencias por un cuadro clínico que había iniciado once días atrás, consistente en pérdida súbita de la visión de ambos ojos, asociado a sensación rotacional que duró aproximadamente siete segundos y que se resolvió espontáneamente; sin embargo, refirió que había persistido la alteración de la visión de manera binocular hacia el lado derecho. Como síntomas relacionados presentó cefalea hemicraneana ipsilateral, de tipo pulsátil, progresiva, de intensidad moderada a severa, asociada a náuseas, emesis, fotofobia, fonofobia, de aproximadamente cinco horas de duración. La mujer tiene antecedentes de cefalea de similares características desde los 16 años, que cumplen con los criterios de migraña con aura y que se presenta alrededor de dos veces al año, por lo que solo recibe manejo sintomático con analgésicos. En el examen físico general no se encontraron hallazgos de importancia; en el examen neurológico se documentó mediante campimetría una hemianopsia homónima izquierda congruente.

Clínicamente, se planteó que la paciente cursaba con un ACV isquémico en el territorio correspondiente a la arteria cerebral posterior derecha. La resonancia magnética (RM) de cerebro mostró un infarto isquémico subagudo que involucraba la corteza medial del lóbulo occipital derecho (figura 1). Se le realizó la estratificación del riesgo vascular en el contexto de ACV en paciente joven.
Los exámenes paraclínicos no mostraron alteraciones relevantes (tabla 2); entre tanto, la venografía por RM cerebral con gadolinio, la angiorresonancia cerebral y el ecocardiograma transesofágico fueron normales, al igual que las pruebas para detección del síndrome antifosfolípidos. En este caso no se realizó panangiografía, por problemas administrativos. Se inició manejo para prevención secundaria con ASA y atorvastatina.

\section{Figura 1.}

Resonancia magnética cerebral simple, cortes axiales secuencias difusión, mapa, FLAIR y T2 coronal ( $A, B, C$ y $D)$ con hiperintensidad en la corteza medial del lóbulo occipital derecho, principalmente en la región de la cuña, con extensión a una pequeña porción de la corteza de la precuña y del giro lingual adyacentes, compatible con infarto isquémico subagudo

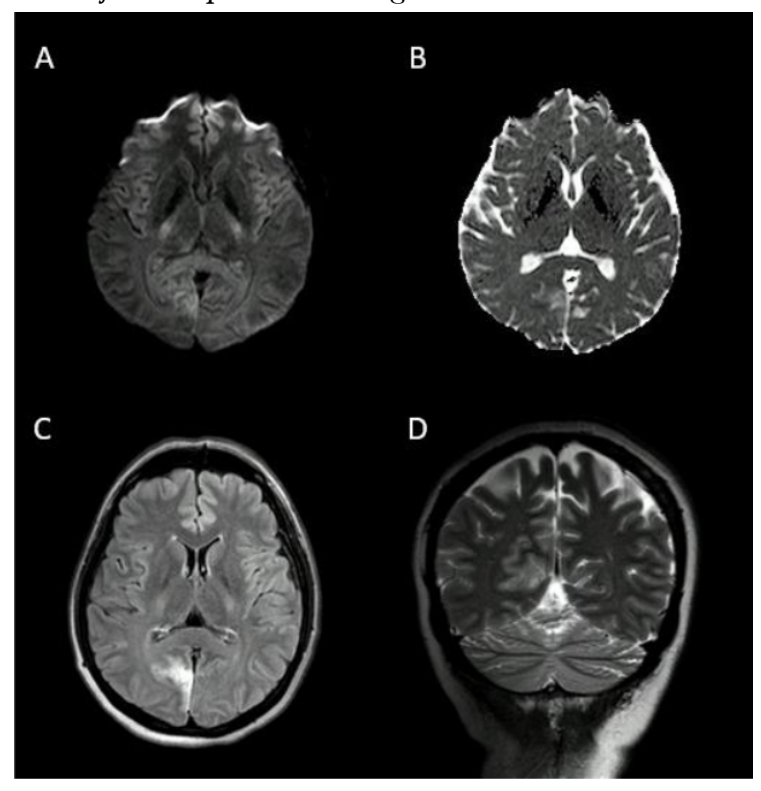


Tabla 2

Exámenes paraclínicos comparativos

\begin{tabular}{|c|c|c|c|}
\hline Laboratorios & Valores de referencia & Caso clínico 1 & Caso clínico 2 \\
\hline \multicolumn{4}{|c|}{ Tiempos de coagulación (s) } \\
\hline PT & Control 11,5 & 11,6 & 11,5 \\
\hline PTT & Control 30,2 & 30,2 & 23,9 \\
\hline INR & & 1 & \\
\hline \multicolumn{4}{|c|}{ Cuadro hemático } \\
\hline Leucocitos & $4,5-10 \times 10^{\prime} 3 / \mathrm{uL}$ & $7,4 \times 10^{\circ} 3 / \mathrm{uL}$ & $13 \times 10^{\prime} 3 / \mathrm{uL}$ \\
\hline Glóbulos rojos & $4.2-5.4 \times 10^{\prime} 6 / \mathrm{uL}$ & $4.7 \times 10^{\prime} 6 / \mathrm{uL}$ & $5 \times 10^{\prime} 6 / \mathrm{uL}$ \\
\hline Hemoglobina & $12,5-16 \mathrm{~g} / \mathrm{d} 1$ & $14,3 \mathrm{~g} / \mathrm{dl}$ & $15,3 \mathrm{~g} / \mathrm{dl}$ \\
\hline Hematocrito & $37-47 \%$ & $41 \%$ & $44,9 \%$ \\
\hline $\mathrm{MCV}$ & $79-101 \mathrm{fL}$ & $86,4 \mathrm{fL}$ & $89,2 \mathrm{fL}$ \\
\hline $\mathrm{MCH}$ & $26-351$ & $30.2 \mathrm{pg}$ & $30.3 \mathrm{pg}$ \\
\hline $\mathrm{MCHC}$ & $31-37 \mathrm{~g} / \mathrm{dl}$ & $34,9 \mathrm{~g} / \mathrm{dl}$ & $34 \mathrm{~g} / \mathrm{dl}$ \\
\hline Plaquetas & $150-450 \times 10^{\circ} 3 / \mathrm{Ul}$ & $336 \times 10^{\prime} 3 / \mathrm{U1}$ & $\begin{array}{l}375,6 \times \\
10,3 / \mathrm{U}\end{array}$ \\
\hline Linfocitos & $20-45 \%$ & $23.3 \%$ & $20.2 \%$ \\
\hline Neutrófilos & $45-70 \%$ & $68,7 \%$ & $69,6 \%$ \\
\hline VSG & $0-20 \mathrm{~mm} / \mathrm{h}$ & $28 \mathrm{~mm} / \mathrm{h}$ & $13 \mathrm{~mm} / \mathrm{h}$ \\
\hline Glucemia basal & $65-110 \mathrm{mg} / \mathrm{dl}$ & $102 \mathrm{mg} / \mathrm{dl}$ & \\
\hline Creatinina & $0,52-1,3 \mathrm{mg} / \mathrm{dl}$ & $0,68 \mathrm{mg} / \mathrm{dl}$ & \\
\hline BUN & $7-20 \mathrm{mg} / \mathrm{dl}$ & $12,8 \mathrm{mg} / \mathrm{dl}$ & \\
\hline \multicolumn{4}{|c|}{ Perfil lipidico } \\
\hline \begin{tabular}{|l|} 
Colesterol total \\
Triogicérics
\end{tabular} & $\frac{140-200 \mathrm{mg} / \mathrm{dl}}{35-160 \mathrm{m \sigma} 1}$ & $\frac{193,2 \mathrm{mg} / \mathrm{dl} 1}{198,3 \mathrm{m \sigma} / \mathrm{d} 1}$ & $200,4 \mathrm{mg} / \mathrm{dl} 1$ \\
\hline $\mathrm{HDL}$ & 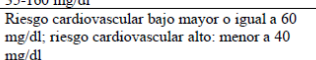 & $\frac{198,3 \mathrm{mg} / \mathrm{dl} \mathrm{l}}{41 \mathrm{mg} / \mathrm{dl}}$ & $61 \mathrm{mg} / \mathrm{dl}$ \\
\hline \multicolumn{4}{|c|}{ Electrólitos } \\
\hline Sodio & $137-145 \mathrm{mmol} / 1$ & $135 \mathrm{mmol} / 1$ & $138 \mathrm{mmol} / 1$ \\
\hline Cloro & $98-107 \mathrm{mmo} / 1$ & $106 \mathrm{mmo} / 1$ & $103 \mathrm{mmol} / 1$ \\
\hline Potasio & $3,6-5 \mathrm{mmol} / 1$ & $3,8 \mathrm{mmol} / 1$ & $3,6 \mathrm{mmol} / 1$ \\
\hline $\begin{array}{l}\text { Acs Anticardiolipina } \\
\text { IGG }\end{array}$ & $\begin{array}{l}\text { Negativo: menor a } 19,9 \mathrm{GPL} \text {; positivo débil: } 20 \text { - } \\
79,9 \mathrm{GPL} \text {; positivo fuerte: mayor de } 80 \mathrm{GPL}\end{array}$ & 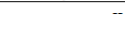 & 4,10 GLPU/M1 \\
\hline $\begin{array}{l}\text { Acs Anticardiolipina } \\
\text { IGM }\end{array}$ & $\begin{array}{l}\text { Negativo: menor a 19,9 MPL; positivo débil: } 20- \\
79,9 \text { MPL; positivo fuerte: mayor de } 80 \text { MPL }\end{array}$ & - & $\begin{array}{r}8,53 \\
\text { MPLU/M1 }\end{array}$ \\
\hline $\begin{array}{l}\text { Beta } 2 \text { glicoproteína: } \\
\text { Acs IgG }\end{array}$ & $\begin{array}{l}\text { Negativo: } 0 \text { a } 20 \text { unidades; positivo: mayor a } 20 \\
\text { unidades }\end{array}$ & - & 0,9 unidades \\
\hline $\begin{array}{l}\text { Beta } 2 \text { glicoproteína: } \\
\text { Acs IgM }\end{array}$ & $\begin{array}{l}\text { Negativo: } 0 \text { a } 20 \text { unidades; positivo: mayor a } 20 \\
\text { unidades }\end{array}$ & -- & 5,4 unidades \\
\hline Anticoagulante lúpico & $\begin{array}{l}\text { Entre } 1,2 \text { y } 1,5: \text { débilmente presente; entre } 1,5 \text { y } \\
\text { 2,0: moderadamente presente; mayor a } 2,0 \text {. } \\
\text { fuertemente presente }\end{array}$ & - & 0,89 ratios \\
\hline
\end{tabular}

Caso 2

El segundo caso corresponde a una mujer de 35 años de edad, natural y procedente de Bogotá, quien ingresó al servicio de urgencias por un cuadro clínico de diez horas de evolución, consistente en una alteración binocular de la visión hacia el lado derecho asociado con cefalea hemicraneana izquierda, tipo peso, de intensidad moderada a severa, asociada a náuseas y fotofobia. Como antecedentes de importancia refirió cefalea tipo migraña con aura desde los 22 años. En el momento de la consulta la frecuencia era de hasta tres veces por semana, sin manejo profiláctico. En el examen físico general no se documentaron hallazgos de importancia. En el examen neurológico, el único hallazgo positivo que se evidenció fue una hemianopsia homónima derecha no congruente.

Se planteó que la paciente cursaba con un ACV isquémico en el territorio de la arteria cerebral posterior izquierda. La RM cerebral simple mostró un infarto isquémico agudo cortico-subcortical en el aspecto medial de lóbulo occipital izquierdo (figura 2). Se le tomaron exámenes paraclínicos de extensión que no mostraron alteraciones relevantes (tabla 2). Se le realizó un ecocardiograma transesofágico, que estuvo dentro de los límites normales; la panangiografía reportó cambios secundarios a una lesión isquémica establecida en el territorio cerebral posterior izquierdo, y el aortograma torácico se encontró sin alteraciones. Dados los antecedentes médicos, la evolución de la paciente y los paraclínicos reportados, se diagnosticó un ACV migrañoso; sin embargo, se entregó orden para estudios ambulatorios de trombofilia y se indicó manejo profiláctico con amitriptilina y control con el servicio de neurología.

\section{Figura 2.}

Resonancia magnética cerebral simple. Cortes axiales secuencias difusión, mapa, FLAIR y T2 coronal $(A, B, C$ y $D)$ que muestran un infarto isquémico agudo cortico-subcortical en el aspecto medial del lóbulo occipital izquierdo que involucra predominantemente la región de la cuña

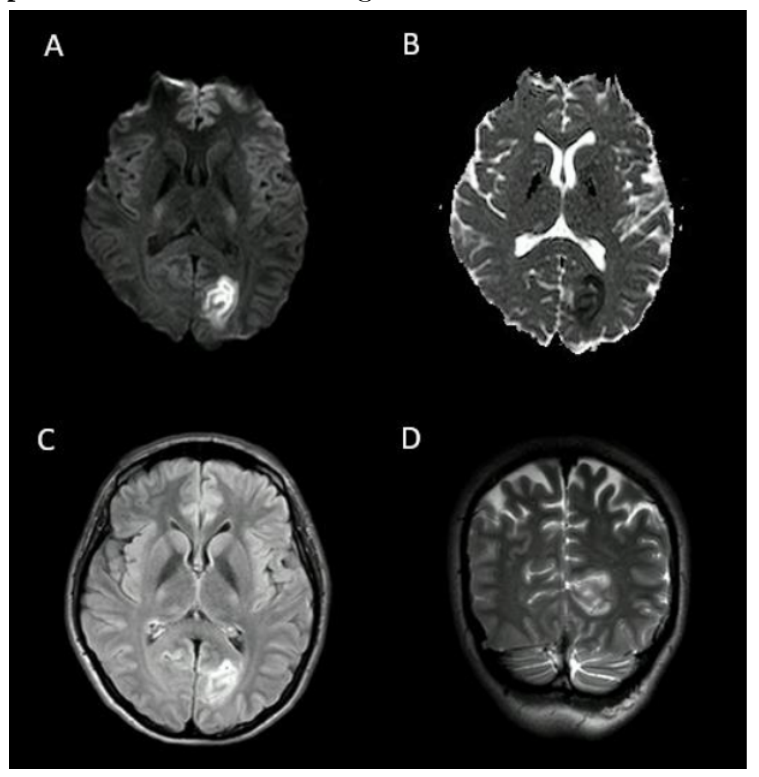

\section{Discusión}

El infarto migrañoso se define como un ACV que se produce durante un episodio de migraña con aura en la que los síntomas del aura persisten durante más de sesenta minutos (4); 
además, se debe demostrar una lesión mediante neuroimagen en el territorio congruente de los síntomas (tabla 1). Por lo general, los infartos son pequeños y se limitan a un único territorio vascular, y se presentan con más frecuencia en el territorio posterior (9).

Se estima que la incidencia anual del infarto migrañoso es de 0,8 casos por cada 100.000 habitantes, tanto para lesiones isquémicas como para las hemorrágicas (10); sin embargo, el ACV isquémico tiene mayor correlación con la migraña con aura que el ACV hemorrágico (5). El infarto migrañoso está catalogado como una complicación de la migraña con aura y representa un reto diagnóstico, ya que las auras prolongadas o atípicas pueden ser difíciles de distinguir de un ACV o de accidente isquémico transitorio, en particular las auras motoras de la migraña hemipléjica (11).

Los factores de riesgo documentados para ACV en pacientes con migraña son: migraña con aura, género femenino, uso de tabaco y estrógenos (12). En algunos estudios revisados, las mujeres con migraña tuvieron un riesgo mayor de presentar un ACV isquémico, con un OR de 2,89 (IC95 \%: 2,42-3,45), comparado con mujeres que no sufrían migraña (13). Adicionalmente, el riesgo es mayor en la migraña con aura, ya que se encontró que el OR del ACV isquémico fue de 2,51 (IC95\%: 1,52-4,14) en pacientes con migraña con aura comparado con un OR de 1,29 (IC95 \%: 1,41-4,97) en aquellos con migraña sin aura (14). Se ha propuesto que el aumento del riesgo de ACV se explica por la presencia de factores de riesgo cardiovascular clásicos, como tabaco, hipertensión arterial o dislipidemia (1).

Actualmente, no se ha demostrado una relación directa entre el número de ataques de migraña y la presencia de lesiones de sustancia blanca, enfermedad de pequeño vaso o ACV (11). La fisiopatología subyacente a la migraña como causa o consecuencia de ACV no está clara; es tanta la incertidumbre que se han descrito múltiples hipótesis de esta posible relación entre las que se incluyen: 1) cambios en el flujo sanguíneo cerebral y vasoespasmo; 2) prolongación de la onda de DCP, que genera inhibición del funcionamiento cerebral; 3) hiperexcitabilidad por glutamato, que aumenta la susceptibilidad a despolarizaciones isquémicas; 4) mayor agregabilidad plaquetaria; 5) liberación de factores protrombóticos o péptidos vasoactivos; 6) anomalías endoteliales, o 7) presencia de polimorfismos genéticos, por ejemplo, el de la enzima metilenetetrahidrofolato reductasa C677T (15-17).

Una de las posibilidades sugiere que la onda de DCP genera una despolarización en masa de la sustancia gris, que resulta en una ruptura de la transmisión neuronal debido a alteraciones en el gradiente iónico, secundaria a hipoxia o isquemia (18). La onda puede ser precedida por un silencio eléctrico que se propaga o no lo hace. Cuando se propaga, se manifiesta clínicamente como un episodio isquémico, y cuando no se propaga, se manifiesta como un episodio de migraña con aura (7). No está claro qué determina la propagación de la onda; sin embargo, se cree que algunos factores genéticos, vasculares y metabólicos como la disección arterial cervical, estados de hipercoagulabilidad, disfunción endotelial y embolismo paradójico explican esta relación (19).

En un episodio isquémico hay depleción de ATP, lo que causa disfunción de la bomba $\mathrm{Na}-\mathrm{K}$ ATPasa. Esto genera pérdida del gradiente iónico y una despolarización sostenida de la membrana celular (7). Asociado a este fenómeno, se presenta una fase de hiperemia extendida de corta duración, seguida de un periodo de oligohemia que persiste durante 1-2 horas y que se cree alcanza niveles de isquemia debido a una vasoconstricción severa (15). Así mismo, la onda de DCP libera moléculas proinflamatorias en el espacio extracelular que inflaman y activan el sistema trigeminovascular, lo cual es suficiente para activar las aferencias meníngeas y causar la cefalea (19).

En cuanto a la localización de los infartos, los investigadores del estudio CAMERA sugieren que la explicación más probable para que las lesiones aparezcan en la zona fronteriza de la circulación posterior es una combinación de hipoperfusión y tromboembolismo local (20). La hipoperfusión y el embolismo, a menudo, 
coexisten y sus características fisiopatológicas son interactivas, debido a que el estrechamiento luminal arterial y las anomalías endoteliales estimulan la formación y, posteriormente, la embolización. Así mismo, la hipoperfusión limita la capacidad del torrente sanguíneo para eliminar los microémbolos y reduce el flujo de sangre disponible, lo que genera isquemia en el tejido cerebral (20).

Hace poco se realizó una cohorte de pacientes en la que se encontró una asociación entre migraña, fibrilación auricular y ACV (8). Según los resultados del estudio, la asociación fue más fuerte en los pacientes que cursaron con migraña y aura. Los autores sugieren que la migraña con aura puede ser una manifestación clínica de microémbolos en el cerebro originados en la aurícula izquierda. Esta teoría está sustentada por un estudio en ratones, en el que inyectaron diferentes tipos de microémbolos en la carótida; posteriormente, los investigadores observaron la onda DCP en el cerebro de los ratones. Además, otros resultados de estudio mostraron que: 1) todos los episodios de DCP estaban precedidos por hipoperfusión global o regional dentro de los 2 minutos previos, 2) la composición y el tamaño de los microémbolos generaron diferentes grados de DCP, 3) la DCP ocurrió en los territorios vasculares más hipoperfundidos y 4) los microémbolos solo generaron microinfartos en el $30 \%$ de los casos (21).

Se han propuesto múltiples mecanismos para explicar la posible relación entre el FOP y la migraña con aura (22). Una de las hipótesis más aceptadas sugiere que un grado de comunicación interauricular más grande se traduce en mayor concentración de hemoglobina desoxigenada, lo que provoca una activación neurovascular y vasorregulación anormal; así mismo, algunos metabolitos, como la serotonina, el óxido nítrico y las cininas, evitan la circulación pulmonar a través del FOP, y hace que entren en la circulación sistémica y causen irritación del nervio trigémino y la vasculatura cerebral (22).

En cuanto a la prevención del infarto migrañoso, no existe evidencia de terapias que disminuyan el riesgo de ACV (23). Las recomendaciones para la suspensión de los anticonceptivos orales son controversiales, debido a que la evidencia se basaba en literatura antigua, en la cual se utilizaban altas dosis de estrógenos; sin embargo, algunos estudios establecen hoy en día que el consumo de anticonceptivos orales con bajas dosis de estrógenos no confiere ningún riesgo adicional. Es más, se ha probado que con una dosis de $20 \mu \mathrm{g}$ de etinil estradiol disminuye la frecuencia de la migraña durante el periodo de la menstruación, al igual que la frecuencia del aura. Adicionalmente, al acortar el periodo libre de hormonas de los anticonceptivos orales ( 24 activo/4 inactivo) hubo una reducción de la frecuencia y la severidad de la migraña, pues se logra una estabilización hormonal $(24,25)$.

\section{Conclusión}

Está claro que hay una relación entre migraña y ACV; no obstante, aún se desconocen los mecanismos fisiopatológicos que existen entre estas dos patologías. Es fundamental tener en cuenta los factores de riesgo que incrementan la probabilidad de sufrir un infarto migrañoso, pues es evidente que esta patología es un reto diagnóstico, por la gran similitud semiológica de ambas entidades. Es importante poder clarificar la relación entre estas dos enfermedades, para aplicar estrategias efectivas de prevención en la población y, así, reducir las complicaciones que conlleva un ACV en este grupo de pacientes.

\section{Conflicto de intereses}

Los autores declaran no tener conflictos de intereses.

\section{Referencias}

1. Mahmoud AN, Mentias A, Elgendy AY, Qazi A, Barakat AF, Saad M, et al. Migraine and the risk of cardiovascular and cerebrovascular events: A metaanalysis of 16 cohort studies including 1152407 subjects. BMJ Open. 
2018;8(3):1-10. https://doi.org/10.113 6/bmjopen-2017-020498

2. Lipton RB, Stewart WF, Diamond S, Diamond ML, Reed M. Prevalence and burden of migraine in the United States: Data from the American Migraine Study II. Headache. 2001;41(7):646-57. https://doi.org/10. 1046/j.1526-4610.2001.041007646.x

3. Lantz M, Sieurin J, Sjölander A, Waldenlind E, Sjöstrand C, Wirdefeldt K. Migraine and risk of stroke: A national population-based twin study. Brain. 2017;140(10):2653-62. https://d oi.org/10.1093/brain/awx223

4. Vincent M, Wang S. Headache Classification Committee of the International Headache Society (IHS): The International Classification of Headache Disorders 3rd edition. Cephalalgia. 2018;38(1):1-211.

5. Magalhães JE, Sampaio Rocha-Filho PA. Migraine and cerebrovascular diseases: Epidemiology, pathophysiological, and clinical considerations. Headache. 2018;58(8):1277-86. https://doi.org/10 $.1111 /$ head.13378

6. Øygarden H, Kvistad CE, Bjørk M, Thomassen L, Waje-Andreassen U, Naess H. Diffusion-weighted lesions in acute ischaemic stroke patients with migraine. Acta Neurol Scand Suppl. 2014;129(198):41-6. https://doi.org/10 $.1111 /$ ane. 12236

7. Oliveira-Ferreira A, Winkler M, Reiffurth C, Milakara D, Woitzik J, Dreier J. Spreading depolarization, a pathophysiologic mechanism of stroke and migraine aura. Future Neurol. 2012;7(1):45-64. https://doi.org/10.22 17/fnl.11.69

8. Sen S, Androulakis XM, Duda V, Alonso A, Chen LY, Soliman EZ, et al. Migraine with visual aura is a risk factor for incident atrial fibrillation: A cohort study. Neurology. 2018;91(24):e2202-10. https://doi.org/ 10.1212/WNL.0000000000006650

9. Wolf ME, Szabo K, Griebe M, Förster A, Gass A, Hennerici MG, et al. Clinical and MRI characteristics of acute migrainous infarction. Neurology. 2011;76(22):1911-7. https://doi.org/10 .1212/WNL.0b013e31821d74d5

10. Kurth T, Winter AC, Eliassen AH, Dushkes R, Mukamal KJ, Rimm EB, et al. Migraine and risk of cardiovascular disease in women: Prospective cohort study. BMJ. 2016;353(June):3411.

11. Fisher CM. Late-life migraine accompaniments as a cause of unexplained transient ischemic attacks. Can J Neurol Sci. 1980;7 (1):9-17.

12. Adelborg K, Szépligeti SK, Holland-Bill L, Ehrenstein V, HorváthPuhó E, Henderson VW, et al. Migraine and risk of cardiovascular diseases: Danish population based matched cohort study. BMJ. 2018;360:k96.

13. Spector JT, Kahn SR, Jones MR, Jayakumar M, Dalal D, Nazarian S. Migraine headache and ischemic stroke risk: an updated meta-analysis. Am J Med. 2010;123(7):612-24. https://doi. org/10.1016/j.amjmed.2009.12.021

14. Schürks $M$, Rist $P M$, Bigal ME, Buring JE, Lipton RB, Kurth T. Migraine and cardiovascular disease: systematic review and meta-analysis. BMJ. 2009;339(7728):1015.

15. Kurth $\mathrm{T}$, Chabriat $\mathrm{H}$, Bousser MG. Migraine and stroke: A complex association with clinical implications. Lancet Neurol. 2012;11(1):92-100. https://doi.org/10. 1016/S1474-4422(11)70266-6

16. Caminero AB, Sánchez del Río González M. Migraña como factor de riesgo cerebrovascular. Neurología. 
2012;27(2):103-11. https://doi.org/10. 1016/j.nrl.2011.03.009

17. Eikermann-Haerter K, Hyun Lee J, Yuzawa I, Liu CH, Zhou Z, Kyoung Shin $\mathrm{H}$, et al. Migraine mutations increase stroke vulnerability by facilitating ischemic depolarizations. Circulation. 2012;125(2):335-45.

18. Dreier JP, Reiffurth C. The strokemigraine depolarization continuum. Neuron. 2015;86(4):902-22. https://do i.org/10.1016/j.neuron.2015.04.004

19. Mawet J, Kurth T, Ayata C. Migraine and stroke: in search of shared mechanisms. Cephalalgia. 2015;35(2):165-81.

20. Kruit MC, Launer LJ, Ferrari $\mathrm{MD}$, van Buchem MA. Infarcts in the posterior circulation territory in migraine. The populationbased MRI CAMERA study. Brain. 2005;128(9):2068-77. https://doi.org/1 $0.1093 /$ brain/awh542

21. Fridman S, Sposato LA. Migraine with visual aura, incident $\mathrm{AF}$, and stroke risk [editorial]. Neurology. 2018;91(24):1077-8. https://doi.org/10 $.1212 / W N L .0000000000006637$

22. Sadrameli SS, Gadhia RR, Kabir R, Volpi JJ. Patent foramen ovale in cryptogenic stroke and migraine with aura: does size matter? Cureus. 2018;10(8):e3213.

23. Gryglas A, Smigiel R. Migraine and stroke: what's the link? What to do? Curr Neurol Neurosci Rep. 2017;17(3):22. https://doi.org/10.1007 /s11910-017-0729-y

24. Faubion SS, Casey PM, Shuster LT. Hormonal contraception and migraine: clinical considerations. Curr Pain Headache Rep. 2012;16(5):461-6. https://doi.org/10.1 007/s11916-012-0278-7

25. Calhoun AH, Batur P. Combined hormonal contraceptives and migraine:
An update on the evidence. Cleve Clin J Med. 2017;84(8):631-8. https://doi.o rg/10.3949/ccjm.84a.16033 